\title{
Protée
}

\section{Les cicatrices cinéma(pho)tographiques des spectateurs cannois}

\section{Damien Malinas et Olivier Zerbib}

Volume 31, numéro 2, automne 2003

Cannes hors projections

URI : https://id.erudit.org/iderudit/008755ar

DOI : https://doi.org/10.7202/008755ar

Aller au sommaire du numéro

\section{Éditeur(s)}

Département des arts et lettres - Université du Québec à Chicoutimi

ISSN

0300-3523 (imprimé)

1708-2307 (numérique)

Découvrir la revue

Citer cet article

Malinas, D. \& Zerbib, O. (2003). Les cicatrices cinéma(pho)tographiques des spectateurs cannois. Protée, 31(2), 63-71. https://doi.org/10.7202/008755ar
Résumé de l'article

Cet article propose d'explorer les traces que laisse le Festival de Cannes dans la mémoire de ses spectateurs au travers des images photographiques que ces derniers produisent, achètent ou "volent ", le temps d'une pause cannoise. Plongées dans un bain cinématographique, ces « mises à l'épreuve » de la réalité festivalière se fixent en cicatrices visuelles qu'arborent les publics, s'incarnent en démarches de spectateurs qui révèlent parfois des pas mis volontairement dans la mauvaise empreinte. L’imaginaire qui se trouve piégé dans les « photographies souvenir » du Festival de Cannes témoigne des moyens que se donne le cinéphile ordinaire de croire et de faire croire à sa participation au monde du cinéma. Cet attachement particulier au Festival de Cannes, réifiant le fétichisme déjà mis en place par le dispositif cinématographique, griffe de sa marque la carrière de spectateurs cannois. 


\section{LES CICATRICES CIN ÉM A(PHO )TO G RAPH IQ U ES DES SPECTATEU RS CAN N O IS}

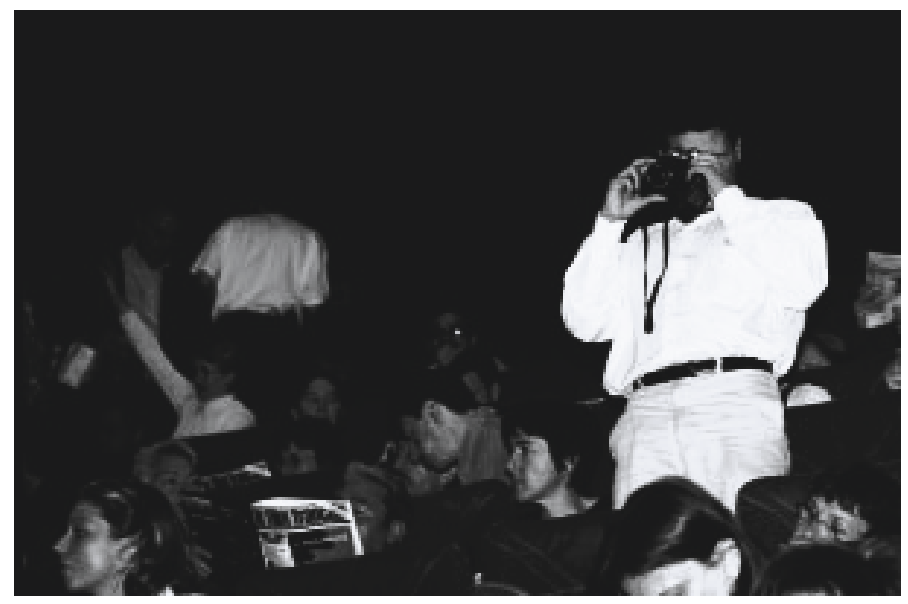

Dans l'illusion, c'est-à-dire la forme la plus courante de mise à l'écart du réel, il n'y a pas à signaler de refus de perception à proprement parler. La chose n'y est pas niée: seulement déplacée, mise ailleurs. Mais, en ce qui concerne l'aptitude à voir, l'illusionné voit, à sa manière, tout aussi clair qu'un autre.

Clément Rosset, Le Réel et son double.

Il n'est pas besoin de se rendre au Festival international du film de Cannes pour savoir que ce qui s'y joue excède largement le très sérieux processus de sélection et de présentation de films inédits. De fait, la plupart des articles qui s'attachent à décrire cette manifestation empruntent tour à tour leurs métaphores au registre religieux, à l'univers des contes de fées, voire, comme le fait Brian de Palma dans son film Femme fatale, à l'imaginaire de l'intrigue policière. Toutes ces métaphores ont ceci de commun qu'elles pointent, selon des modalités différentes, un des éléments constitutifs du Festival de Cannes: l'essentiel de ce qui s'y passe n'est pas donné à voir au public. Élisabeth Claverie commente cette analogie, souvent constatée entre la sacralité religieuse et celle dont semblent empreintes les manifestations cannoises, en précisant que l'on retrouve dans les deux cas «le 
même processus de séparation, de discipline des regards, de difficulté d'assignation des provenances» ${ }^{1}$. C'est grâce à ce principe que ce qui est initialement un dispositif professionnel destiné à assurer la promotion et la valorisation d'œuvres cinématographiques sélectionnées ${ }^{2}$, en somme une activité très austère, peut devenir une manifestation empreinte de magie. Pour qu'il y ait "magie» ${ }^{3}$, il faut de l'inconnu et du spectaculaire: le prestidigitateur détourne notre attention de son «truc" par les paillettes et les mensurations de son assistante:

Quelqu'un qui dirigera sous nos yeux une aiguille de façon convaincante nous fera entrevoir un fil qui n'existe pas [...], les illusionnistes professionnels sont capables de faire apparaître ces perceptions fantômes. Ils nous amènent à suivre, selon un déroulement préou, des situations qui nous sont apparemment familières, qui font que notre imagination nous entraîne et comble d'elle-même les lacunes, sans que nous puissions savoir à quel moment nous avons été trompés. 4

À Cannes, les nombreux événements qui encadrent la projection d'un film (montée des marches, présence des acteurs, des réalisateurs ou des producteurs, fêtes et réception en tous genres, etc.) détournent l'attention des participants, du fait qu'ils ne prendront pas une part active aux délibérations $\mathrm{du}$ jury sanctionnant le palmarès, et offrent aux regards un spectacle savamment mis en scène. La poudre aux yeux cannoise appartient à ce que Cocteau appelle "un mensonge qui dit la vérité»: si ce spectacle s'exhibe aux yeux de tous, la place de chacun y est clairement assignée. Tout accrédité se félicite de pouvoir accéder à certains des lieux dans lesquels les événements qui rythment le Festival de Cannes se déroulent, mais on ne peut oublier que cette même accréditation lui interdit l'accès à bien plus d'espaces qu'elle ne lui en autorise. En dispensant les privilèges, l'organisation du festival laisse penser aux participants, qu'ils soient professionnels éclairés ou simples badauds de passage, qu'ils se rapprochent du cour du dispositif cannois tout en se donnant les moyens de gérer leur mise à distance. À l'image de ce qui se produit dans une société de Cour, l'existence d'un apparat réglé protocolairement autorise et légitime l'exercice d'un pouvoir fortement dissymétrique, pouvoir qui récompense l'allégeance en créditant ses vassaux d'un rang dans un système de privilèges. Alors que l'on accède à la cour cannoise en faisant montre de son appartenance au «monde du cinéma", le fait d'être ou non accrédité renforce, affaiblit ou annule cette appartenance et le rôle de prescripteur que l'on peut endosser sur son propre territoire culturel et géographique.

Si les aspects «spectaculaires» du Festival de Cannes nous renseignent sur ce que ces publics viennent y faire et, par extension, sur ce qu'ils font durant l'année, il faut considérer que les photographies qui y sont produites constituent un matériau de premier choix pour étudier les attitudes spectatorielles hors projections. Beaucoup de commentateurs du fait cannois ne s'y sont d'ailleurs pas trompés en s'intéressant de près aux clichés de stars et à la fétichisation de celles-ci par les cinéphiles. Mais Cannes, on le sait, ne se limite pas à l'activisme des publics de ces premiers cercles (organisateurs, réalisateurs, acteurs, producteurs y défendant leurs films ou professionnels accrédités à titres divers), puisque ceux que l'on a qualifiés de spectateurs du troisième cercle ${ }^{5}$, participants anonymes mais néanmoins actifs présidant avec force à la destinée d'une œuvre filmique au-delà de la temporalité cannoise, concourent à donner au Festival international du film une tonalité qui le distingue de ses homologues berlinois ou vénitien, plus professionnalisés.

De fait, le festival vit également par l'activité de ces spectateurs du troisième cercle, tour à tour spectateurs et acteurs de la mise en scène cannoise. Sans leurs regards, un événement tel que la montée des marches ne pourrait revêtir un aspect cérémoniel, ne s'apparenterait à rien d'autre qu'à une séance de photos privée, telle qu'il s'en produit tout au long de l'année. Sans leurs silhouettes anonymes formant l'arrière-plan des photographies de stars, nul ne pourrait mesurer l'écart censé distinguer ces dernières de la foule ${ }^{6}$. Aux côtés des barrières triant la foule des 
accrédités, des marches du palais, du tapis rouge ou de la masse agglutinée des photographes, la présence de ces publics est un élément conventionnel des codes de représentation photographique ou télévisuelle du Festival international du film. On peut d'ailleurs constater que, lors d'un autre temps fort de cette manifestation, la cérémonie de clôture durant laquelle se déroule la remise des prix du palmarès, ces codes cessent d'être nécessaires pour désigner le contexte cannois: les stars qui sont prises en photos montrent ostensiblement leur trophée aux photographes, symbole déictique du festival: ceci est le Festival international du film de Cannes. En s'appropriant ces codes, en les incorporant et en en jouant à l'aide de photographies réalisées, achetées ou, le cas échéant, volées, les festivaliers ordinaires parviennent à domestiquer le dispositif cannois, apprennent à s'y repérer et à y définir leur propre rôle, se donnent, enfin, les moyens de rapporter cette expérience à ce qui fait leur quotidien de cinéphiles.

\section{L'INCARNATION DU DISPOSITIF CANNOIS EN IMAGES PHOTOGRAPHIQUES}

Prises dans un cadre cannois, les photographies de stars ou d'anonymes ont ceci de particulier qu'elles font très souvent jouer à l'arrière-plan le rôle de référent principal, d'élément qui, entre tous, est porteur de sens. Au sein de l'histoire du festival et par l'accumulation de leurs histoires, ce sont les stars qui ont conféré à la représentation de ces lieux ce statut de référent photographique. C'est pour avoir maintes et maintes fois vu ces lieux en arrière-plan des photos de stars que l'on sait qu'il s'agit de Cannes. Or, aujourd'hui et depuis un certain temps, c'est en voyant des individus sur cet arrière-plan que l'on peut savoir qu'il s'agit de stars, même si on ne les connaît pas. La représentation photographique d'individus sur cet arrière-plan, le décorum cannois, leur accorde une photogénie cinématographique en même temps qu'elle leur alloue ce que l'on pourrait appeler une cinégénie photographique: les photographies cannoises les désignent comme appartenant au monde du cinéma et, pour être cannoises, ces photographies doivent être cinématographiques. Les spectateurs du troisième cercle jouent de cette ambiguité, de cet espace de représentation qui leur est laissé. À travers cette notion de jeu, du saisissement de cette scène, peut-on décortiquer autrement la production photographique cannoise "amateur» que comme étant uniquement constituée de photographies «signaux» 7 ou, en d'autres termes, de photographies construites par quelqu'un qui sait et qui les utilise pour faire "savoir»?

En effet, une grande partie des photographies produites par les spectateurs eux-mêmes est échafaudée pour "montrer", comme tout un chacun saurait le faire en vacances. Comme les Caroline à la plage et OuiOui s'envole ${ }^{8}$, ces photos mettent et «montrent" en situation: elles rentrent dans la classe des photos à vertu explicative. Qui ne se souvient pas d'une séance «diapo-vacances» d'un pays que l'on ne connaît pas et dont on nous explique qu'on ne peut pas comprendre si l'on n'y est pas allé (alors, pourquoi cette séance diapo?), eh bien, ici, il s'agit de la même chose: «je vais vous expliquer Cannes». Ce sont donc bien des photos «signaux» qui mettent en place un faire-savoir pédagogique. Dans ce faire-savoir, les photographies restituées à Cannes font preuve d'une ambiguïté sur le registre auquel elles appartiennent: relèvent-elles du procédé documentaire ou bien de la formule fiction? À Cannes, on joue à... mais c'est bien vrai qu'on joue à...! On peut certainement rapprocher cette ambiguité de celle qui existe entre la carte postale touristique et les photographies que les touristes produisent en se faisant prendre dans le même cadre que chez le «marchand de souvenirs». Pourquoi préférer ces photos finalement économiquement moins intéressantes et souvent d'une piètre qualité par rapport à la carte postale soignée, qui, de plus, a le mérite de ne pas dévoiler leurs tenues de vacances? Marc Angenot situe justement la transaction avec les êtres vivants à l'œuvre sur ces photos ${ }^{9}$ : la seule question de la personne qui a pris la photo met en jeu des liens, du hors champ qui a été vu (ici, le photographe peut être officiel). Les photos soustendent et authentifient le récit par rapport aux cartes 
postales: les événements qui sont pris en photo ont bien été vécus, non seulement à cet endroit, mais aussi à un moment précis. C'est ce que Roland Barthes souligne en déclarant que

[...] la photo possède une force constative, et que le constatif de la Photographie porte, non sur l'objet, mais sur le temps. D'un point de vue phénoménologique, dans la photographie, le pouvoir d'authentification prime sur le pouvoir de représentation. 10

Reste alors à analyser les photos des photographes professionnels, «confectionneurs» de souvenirs, qui ont pour objectif photographique la vente de leurs clichés, et qui signent leur acte par la remise de leur carte de "spécialiste en photos de stars»" 11 ? Il semble, dans un premier temps, que ce soit une évidence: la mise en scène est flagrante (jusqu'au photographe qui est en smoking); ces photos seraient donc celles qui présentent le plus de «signalité» dans le dispositif cannois. Seulement, cette évidence reste inébranlable à condition de restreindre le cliché photographique à son usage finalisé - «après avoir été acheté, être montré»-, car lorsqu'on s'intéresse au trajet pratique de ces photos, leur constitution en tant que photographies «signaux» n'est plus aussi évidente. Ces photographies "professionnelles" ne sont pas des photographies «signaux» tant qu'elles n'ont pas objectivé leur saisie photographique, et ce, au cours d'un processus. Ces photos «non objectivées» - que l'on peut aussi qualifier "d'indices" - se définissent ainsi, car elles se retrouvent précipitées dans une masse de clichés (que certains pourraient qualifier d'indifférenciés), et sont signalées souvent comme «les photos du lendemain".

Il faut tout de suite remarquer que lorsqu'on les trouve le lendemain dans les stands de présentation, ce sont déjà des photos de la veille. Cet espace d'étal des photographies peut être présenté comme celui de l'indifférenciation, cela n'empêche pas qu'il soit aussi celui de la reconnaissance. Certes les photographies de Catherine Deneuve, Liv Ulman et Gérard Depardieu sont comme, et parmi, celles des «autres" (ceux qui sont allés à Cannes mais qui n'appartiennent pas au monde professionnel du cinéma) présentées sur des éventaires et indexées par la date, l'horaire et la séance du film; seulement, le principe de ces stands est de reconnaitre des stars, de se reconnaître «soi parmi les stars», mais aussi «soi à la place des stars». En distinguant ces photos sur le stand, en se reconnaissant, on les révèle momentanément en tant que photographies «signaux». De fait, l'achat de ces photos stabilise ce statut: c'est une transformation par la reconnaissance de photos «indices» en photos «signaux». C'est peutêtre ici que l'on peut prendre le mieux, outre la dimension communicationnelle des photos cannoises, leur mesure identitaire. Dans ce processus que nous venons de décrire, à qui signale-t-on? On s'adresse à soi, on signale une intimité cinématographique à son identité. C'est ce qui est en jeu plus spécifiquement dans les photographies cannoises, et c'est pourquoi elles ne peuvent être simplement considérées comme des photos de vacances. Il est évident, dans ce que nous avons voulu décrire, que toutes les photographies prises par des amateurs ne sont pas des photos de vacances à vertu explicative et que toutes les photos prises par des spécialistes ne renvoient pas forcément et seulement à l'intimité. Mais cette démarche descriptive s'inscrit dans la nécessité de pointer, en même temps que la diversité de ces photos représentant des «amateurs», leur spécificité.

Quoiqu'il en soit, il y a une chose qui continue à distinguer photos de stars des photos d'anonymes, c'est le relatif isolement que la célébrité autorise: lorsqu'on prend en photo des stars lors de la montée des marches, on interrompt le cheminement, en principe continu, de cette ascension. Dans cet isolement, il faut lire la nécessité de lisibilité des stars; car, comme nous l'avons vu, elles ne sont pas le référent principal de la photographie, elles sont le surligneur qui vient rappeler le sens de cette montée des marches. Les stars sur les photographies cannoises doivent être discrétisées, on doit pouvoir détacher leur unité de sens et de forme du flot visuel cannois.

Les spectateurs du troisième cercle ont appris à se discrétiser photographiquement au moyen d'un expédient «temporel» qui fait que lors de la descente 


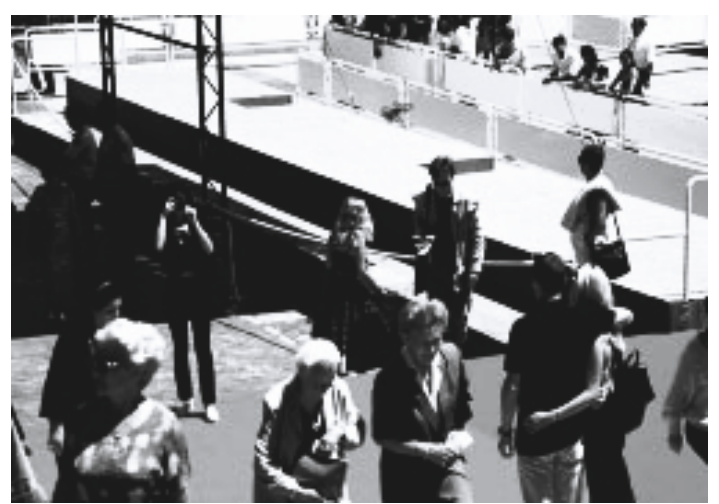

Photo 1 : Mise en scène des festivaliers devant le Palais des festivals

des marches, il est possible de réaliser des

photographies dans les mêmes conditions que les stars: le photographe est officiel et l'uniforme est le même pour tous - tenues de soirée. En effet, à Cannes, comme dans les sociétés de cour et d'apparat, à chaque heure de la journée sa tenue; c'est peut-être là un des ingrédients de l'iconicité photographique cannoise: le paraitre veut dire quelque chose. Ici se trouve une véritable différence entre le festival dans son extériorité montrée et le pèlerinage qui se vit dans une intériorité revendiquée: on ne ramène pour témoigner de son expérience que très rarement des photos de Lourdes ou de Fatima, alors que ramener des photos de Cannes fait partie du témoignage cannois. Si Cannes a à voir avec la religion, c'est plus certainement du côté des cérémonies de mariage qu'il faut situer ce rapport. Des espaces de «photograbilité» des corps en posture s'y dessinent de la même façon: des lieux de pauses et de tenues conventionnelles et obligatoires près du temple aux images festives, qui semblent plus détachées de la notion de mariage, dans un cas, et de l'idée d'un festival de cinéma, dans l'autre, mais qui pourtant sont consubstantielles à ces événements. Il s'agit de rappeler que, dans ces deux cas, les photos qui sont produites en de telles circonstances représentent une identité forte de soi, et que nous ne les montrons pas toutes à tout le monde et à n'importe quelle occasion.

Les actes photographiques des festivaliers - qu'ils relèvent de la pose, de la pause, de la collecte ou de la restitution - nous amènent plus particulièrement à interroger les identités individuelles des spectateurs et l'idée qu'ils se font d'être un public à Cannes; plus généralement celle qu'ils se font d'un public de cinéma. Il est remarquable que les photos cannoises ne saisissent que rarement des groupes de spectateurs. Il n'y a pas, à Cannes, de clichés qui pourraient s'apparenter aux photos de classe, de colonies de vacances, aux photos qui font primer l'existence du groupe comme identifiant social. L'individu ou le couple est placé au premier plan des photos cannoises pour rappeler les mises en scène des stars ou des couples de stars. Certes, la foule cannoise est aussi photographiée et montrée, cependant elle ne renvoie plus à l'identité de spectateur mais à celle de public, elle n'est qu'un cadre de référence. Dans une certaine mesure, ces photographies, par le biais d'une inter-iconicité avec les clichés cinématographiques dont nous avons parlé plus haut, jouent le rôle de référent-décor (notion que nous développons plus loin) dans le récit que l'on peut faire de soi à Cannes. S'intéresser aux photos des spectateurs cannois, ce n'est donc pas éluder la question des publics, mais s'attaquer à son double corps, non seulement celui de spectateur mais aussi celui de sa conscience du public en tant que public ${ }^{12}$.

\section{ÉPREUVES PAR L'IMAGE:}

\section{LA PRODUCTION PHOTOGRAPHIQUE CANNOISE}

Avant l'enquête ${ }^{13}$ sur les publics du festival, les spectateurs du troisième cercle constituaient presque l'impensé du dispositif cannois: relégués au rang de simples témoins des cérémonies cannoises ou, au mieux, d'éléments de décors situés dans l'arrière-plan des photos de stars (ce que nous désignons sous le terme de «référent-décor»); ces spectateurs du troisième cercle étaient rarement regardés comme des agents actifs (et réactifs) indispensables à la réussite de l'alchimie cannoise. Pour les photographes accrédités, en effet, seules les stars comptent et les photographies de spectateurs ne constituent bien souvent qu'un pisaller: elles présentent le seul intérêt d'être monnayables le lendemain dans les boutiques et les stands prévus à cet effet. L'accueil (illustré en un condensé quelque peu «rude» par la photographie 2), qui a été fait par ces mêmes photographes à PierreLouis Suet ${ }^{14}$, illustre bien cette incapacité à considérer les spectateurs du troisième cercle comme des individus qualifiés pour être photographiés de façon officielle. 


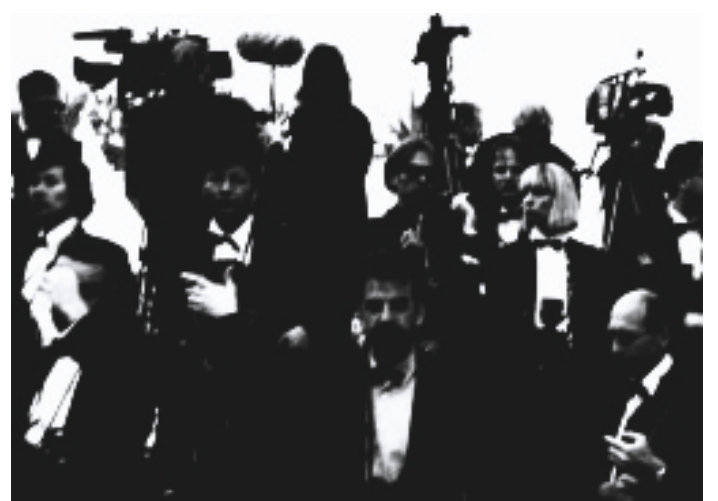

Photo 2: Honneur fait à un photographe des publics

Or ces publics se font une image toute autre de leur rôle et cherchent à palier ce «déficit d'image» par leurs propres moyens. Le sentiment d'appartenir de plein droit au public cannois, et donc par extension au monde du cinéma, aboutit à la nécessité d'avoir recours à la photographie pour rendre palpable cette réalité vécue. Il ne s'agit pas ici de chercher à légitimer sa présence à Cannes en en produisant les preuves ni à proprement parler d'un besoin de reconnaissance qui se traduirait par la mise en place de simulacres destinés à imiter le ballet des stars et des photographes; ce dont il est question concerne des mécanismes identitaires intimes, qui s'adressent avant tout à l'individu lui-même et non pas aux autres. En d'autres termes, faire preuve de sa présence à Cannes, c'est d'abord s'affronter soi-même au dispositif cannois, y évaluer son rôle et sa place et non chercher à en convaincre autrui, sur place ou une fois de retour chez soi. La production ou l'achat de photographies par les spectateurs du troisième cercle ne répond donc pas tant à une frustration qu'à l'affirmation d'une jouissance à être présents en des lieux qui ne leur sont pas directement destinés.

Produire des photographies à Cannes engage donc une série de mécanismes identitaires, dont le premier pourrait se résumer au fait de devenir festivalier. Comment, en d'autres termes, rendre palpable, effective, une identité fondée sur l'illusion cannoise? Comment saisir photographiquement un fantasme? Comment prendre ses désirs pour des réalités? Il ne nous paraît pas inutile ici de nous arrêter un instant sur la phrase de Clément Rosset, en exergue à cet article: «Mais, en ce qui concerne l'aptitude à voir, l'illusionné voit, à sa manière, tout aussi clair qu'un autre». De fait, les photographies prises par les «illusionnés» cannois enregistrent bien la «vision» (à prendre au sens propre, mais également en tant que représentation, même en tant qu'hallucination) que ces derniers ont du festival, vision bien entendu orientée par leur positionnement sur l'échiquier cannois.

Éprouver sa présence à Cannes passe, pour un "accrédité», par la reconnaissance et l'appropriation des codes cannois évoqués plus haut, reconnaissance et appropriation que la photographie contribue à la fois à autoriser et à signaler. En posant, en s'accordant le temps d'une pause cannoise, on cherche à s'adapter, à se conformer à l'image de ce que l'on attend d'un festivalier. Ces photographies s'accumulent comme autant de traces des multiples manières par lesquelles les festivaliers se soumettent à un usage, à une destination, afin de parvenir à pénétrer dans les salles obscures dans lesquelles seront projetés les films des différentes sélections cannoises. Le port du smoking ou de la robe de soirée n'est que la manifestation la plus visible, mais les photographies des spectateurs du troisième cercle témoignent également d'une sorte d'assurance tranquille, cette conviction d'avoir une place dans le dispositif, indispensable au franchissement des barrières, qui peut se muer en pouvoir de persuasion face à la méfiance des vigiles qui gardent les entrées du Palais du festival.

On rassemble donc les photos de soi, mis en scène dans un cadre prévu pour d'autres, sans toujours mesurer l'écart qui sépare ces photographies de celles représentant les stars devant le même décor. Par ailleurs, parvenir à réaliser soi-même des photos d'acteurs ou de réalisateurs montant les marches répond au même besoin de se dire «j'y suis» ou «j'y étais», «je suis capable d'y être, qualifié pour y être».

Mais la domestication du Festival de Cannes suppose également que les accrédités trouvent un sens au fait d'être festivalier, parviennent à faire entrer leur expérience cannoise dans une perspective qui survive à leur retour à la vie quotidienne. Pour exceptionnelles qu'elles soient, les projections cannoises doivent pouvoir prendre place aux côtés des films visionnés ailleurs, afin d'alimenter l'expérience des cinéphiles temporairement devenus festivaliers. Lorsque les événements cannois s'éloignent trop de ce qui fait le quotidien d'un cinéphile, lorsqu'ils 
échappent à ce qui fait le droit commun d'un amateur de cinéma, ils sortent du cadre d'interprétation du film vécu hors projection pour se muer en une expérience sociale et culturelle d'un autre genre, comme une expérience de notabilité culturelle dans le cas des accrédités fréquentant plus les fêtes que les salles de projection. Au contraire, les photographies qui mettent en scène les festivaliers aux côtés des stars ou, le plus souvent, devant les stars (celles-ci pouvant être dans bien des circonstances "capturées" dans un lointain arrière-plan) permettent de faire descendre ces dernières de leur piédestal, de leur redonner une apparence moins intimidante, bref de banaliser quelque peu les manifestations cannoises. On rencontre par ailleurs des photographies qui, bien que formellement différentes, répondent au même effort de domestication: Cannes offre en effet la rare occasion d'observer les stars au quotidien, par exemple attablées à une terrasse de café ou même en train de faire leurs courses. Le fait de pouvoir photographier Julie Delpy faisant la queue dans un supermarché redonne un corps à celle qui pouvait passer pour une apparition dans le tumulte de la montée des marches ou sur l'écran du grand théâtre Louis Lumière. Hors des décors cannois balisés, ceux qui appartiennent au monde du cinéma s'offrent aux photographes amateurs en «référent des corps» (par opposition, on l'aura compris, au terme de «référent décor» utilisé plus avant), leur permettent de garder à l'esprit le fait qu'ils sont comme eux et non des êtres surnaturels, évanescents.

On nous objectera sans doute que ce type d'expérience est précisément autorisé dans le cadre d'un festival tel que celui de Cannes et non dans les salles normales de cinéma où les acteurs restent figés à l'état de «spectres». Il serait alors impossible d'affirmer que ces photographies signalent une mise à distance du dispositif cannois. Bien au contraire, elles témoignent d'une autre de ses particularités, l'opportunité de côtoyer des stars. Cependant, la mise à distance de la magie cannoise contenue dans ces prises de vues nous paraît déterminante, puisqu'elle place ceux qui les produisent dans une situation proche de celle que connaissent les cinéphiles en sortant d'une projection durant l'année, lorsqu'ils cherchent à comprendre, à mettre en mots, les effets que le film a eu sur eux.

En effet, c'est dans ce type de démarche que s'incarne le mieux l'activité des publics cannois, cette participation enthousiaste qui ne fait pas forcément l'économie d'une prise de distance respectueuse, bref cette attitude que l'on rencontre précisément chez les cinéphiles les plus fervents lorsqu'ils recherchent, dans la lecture des critiques, dans les débats entre amis partageant la même passion, les moyens d'aimer le cinéma tout en restant exigeants. On notera d'ailleurs combien les interruptions prolongées ou définitives d'une pratique culturelle surviennent souvent chez les participants les plus assidus, ceux qui ne parviennent plus à en jouir pleinement, qui tiennent à ne pas se montrer naïvement passionnés, mais plutôt informés, critiques ${ }^{15}$.

À cannes, la photographie permet donc également de mettre en doute ce qui est donné à voir et, de ce fait, témoigne d'une maitrise des codes régissant le dispositif festivalier. Tous les clichés mettant en scène les sosies de stars (qui y sont, on le sait, légion sur la Croisette), ou les anonymes présentant une ressemblance cocasse ${ }^{16}$ avec tel ou tel personnage, influent du monde du cinéma, interrogent le statut médiatique des acteurs. Il ne suffit pas d'être présent sur la toile de fond cannoise, sous les projecteurs et dans une tenue de soirée, pour appartenir durablement au monde du cinéma, puisqu'il est possible, pour n'importe qui, d'en faire autant, le temps d'un festival. En jouant avec les codes cannois, les spectateurs du troisième cercle montrent qu'ils peuvent à la fois croire et ne pas croire à la magie du cinéma: ils sont fascinés et en même temps conscients de la part de chance et de travail qu'il faut aux acteurs pour s'imposer durablement sur la scène médiatique. On trouve d'ailleurs, parmi ceux qui ne maîtrisent pas ces codes cannois, des spectateurs pris au piège du rêve cinématographique, sosies taillés au scalpel ou starlettes en quête de producteurs, butant bien souvent au pied des marches du Palais des festivals. 
Ces personnes recherchent à Cannes ce qui n'y est jamais délivré: bien plus qu'une identité de spectateur ou de cinéphile, une identité sociale qui se confondrait avec la fiction cinématographique dont le festival fait étalage.

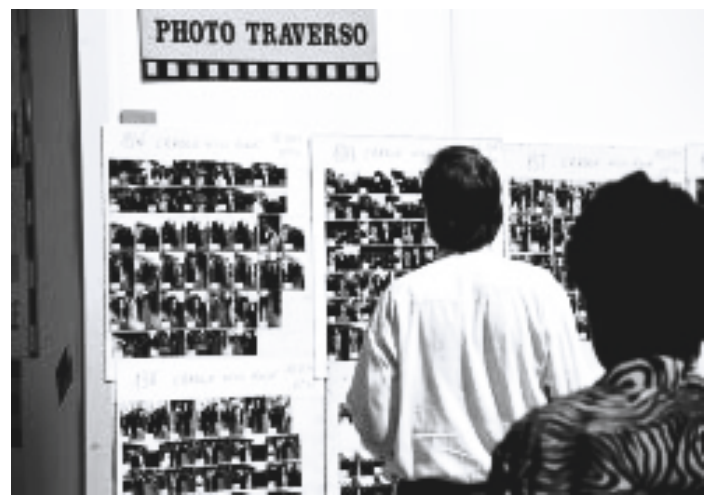

Photo 3: Festivaliers à la recherche de leur double cannois

Il est vrai que les photos cannoises renvoient, et ce, de plus d'une manière, au fétichisme dans sa dimension identitaire ou, plus précisément, de construction de l'identité. Le dispositif cinématographique, en lui-même, met en place une relation fétichiste à l'image, à l'altérité, au miroir. Christian Metz décrit le passage d'une croyance initiale à son désaveu: nous sommes en tous points identiques, mais nous découvrons un jour la différence comme une ruse ${ }^{17}$. Comme spectateurs de cinéma, nous sommes également exposés au désaveu, nous croyons tous assister au même film, et cependant nous découvrons que nous n'y voyons pas tous la même chose. De la même façon, le Festival international du film de Cannes est censé réunir tous ses participants dans une communauté spectatorielle de pensée et de vision autour du cinéma; pourtant, à bien y regarder, la mécanique même de la forme festivalière fait ressortir un rapport singulier au cinéma. Même pour les plus professionnels des spectateurs, il faut bien constater que les critiques n'arrivent que difficilement à accorder leurs violons autour de réceptions définitives des films. Cannes devient alors le lieu d'un rapport singulier qui, malgré une proximité réelle au cinéma, n’arrive pas à dégager de consensus.
Les photos permettent de raconter Cannes par les conventions des représentations qu'elles délivrent: elles deviennent un diapason iconique du discours commun cannois en rendant compte de ce juste-avant où nous étions tous "pareils»: non pas des stars, mais des participants au monde du cinéma. Les festivaliers cannois ne sont pas pour autant naifs, bien qu'ils s'illusionnent à travers ces photographies qu'ils exhibent comme des cicatrices. Mais, outre la dimension exhibitionniste de ces cicatrices, ces photographies sont avant tout un outil de l'énonciation de ce que peut être Cannes, de ce dont on peut en témoigner. Il y a certes la dimension probatoire de ces clichés qui sous-tendent plus particulièrement le récit que les images de magazines (comme nous l'avons noté plus avant en situant les photographies de vacances par rapport aux cartes postales), mais ces épreuves photographiques possèdent une véritable qualité de médiation, elles permettent au tiers écoutant de pouvoir se mettre dans certaines conditions de réception du témoignage. Renaud Dulong décrit le dispositif du témoignage dans la mesure où celui-ci

[...] ne transmet pas un affect, il ranime une disposition à être affecté. Ce qui est en jeu dans la réception ne relève pas d'un procès de diffusion mais du réveil d'un intérêt potentiel déjà présent. 18

En effet, le témoignage cannois, et la "monstration" photographique qui l'étaye, ne peut relever que de la communication affective pour quelqu'un qui éprouve un intérêt positif, critique ou carrément négatif susceptible d'être animé, réanimé pour qu'il y ait une communication effective. Il n'est d'ailleurs pas rare de rencontrer des communautés de spectateurs cannois «hors festival». Nous entendons ici que les membres qui se fréquentent autour du festival, se racontent leurs expériences, se montrent leurs photos, mais ne séjournent pas ensemble lors de l'événement cannois. Ces lieux d'échanges communicationnels, qui prennent comme éléments de discours les clichés cannois, relèvent de ce que met en place une des premières scènes de L'Arme fatale 2, 
lors de la rencontre entre Mel Gibson et René Russo: faisant preuve d'expériences communes mais singulières de combat, l'un et l'autre vont raconter leur carrière de combattant en montrant à la fois leur corps et leurs cicatrices. S'agissant d'un raccourci pour raconter un soi cinématographique en tant que spectateur, mais aussi en tant qu'acteur d'un des moments intenses de la vie du monde du cinéma, les photos restituées du Festival de Cannes rentrent dans le régime des cicatrices cinématographiques qui sont énoncées pour «dire» une identité selon un mode événementiel, qui, de plus, permet de raconter ce que l'on doit faire pour ranimer un affect cannois chez l'autre.

Les photographies qui sont produites par les festivaliers cannois, combinant les aspects documentaires du témoignage et ceux cinématographiques du récit d'événements vécus comme une fiction, constituent ce que nous appellerons finalement des cicatrices "cinéma(photo)graphiques», exemple parmi d'autres de la faculté qu'ont certains usages sociaux d'amoindrir les frontières censées exister entre différents médias.

\section{NOTES}

1. É. Claverie, "Cannes et Chicanes: "Voir à Cannes?" ", dans E. Ethis (sous la dir. de), Aux marches du palais: le Festival de Cannes sous le regard des sciences sociales, Paris, La Documentation française, 2001. 2. Cf. l'article premier des statuts de l'Association française du Festival international du film : «Le Festival de Cannes a pour objet, dans un esprit d'amitié et de coopération universelle, de révéler et de mettre en valeur des œuvres de qualité en vue de servir l'évolution de l'art cinématographique et de favoriser le développement de l'industrie du film dans le monde».

3. Voir à ce propos E. Ethis, «Aux fondements du pacte spectatoriel: les publics de la magie», Revue Les Enjeux («Po(i)étique du questionnaire, spectateurs et publics de la culture réinventés par les enquêtes de pratique»), Grenoble, mai 2003.

4. E.H. Gombrich, L'Art et l'Illusion. Psychologie de la représentation picturale, Paris, Phaidon, 2002, p. 172.
5. E. Ethis, «Introduction. Festival, festivaliers, "festifs-alliés”?», dans E. Ethis (sous la dir. de), Aux marches du palais, op.cit.

6. Voir à ce propos E. Morin, "Le Temps des stars, Dieux et déesses", Les Stars, Paris, Le Seuil, coll. «Points essais», 1972, p. 36-54.

7. Pour une définition plus complète des termes «photographies signaux " et "photographies indices" utilisés dans cet article, voir L. J. Prieto, «Entre signal et indice: l'image photographique et l'image cinématographique», Xoana, n¹, Paris, 1993.

8. Caroline et Oui-Oui sont à la fois le titre de séries d'histoires et le prénom de héros pour enfants, dont la particularité qui nous intéresse ici est de mettre en scène des situations diverses de manière toujours très explicative, presque scolaire.

9. M. Angenot, Critique de la raison sémiotique, Québec, Presses de l’Université de Montréal, 1985.

10. R. Barthes, La Chambre claire, Paris, Gallimard-Le Seuil, 1980, p. 138-139.

11. On peut remarquer que ce genre de pratique photographique professionnelle existe habituellement en période estivale dans les stations balnéaires.

12. Voir autour de cette notion et des débats qu'elle suscite: D. Dayan, "Le double corps du spectateur ", dans J. Bourdon et F. Jost, Penser la télévision, Paris, Nathan, 1998.

13. E. Ethis (sous la dir. de), Les Publics du Festival de Cannes, rapport d'enquête, Paris, Centre national de la cinématographie, 1999.

14. Précisons que P.-L. Suet, membre du laboratoire Culture et Communication chargé de réaliser des clichés des publics composant l'assistance des projections officielles, disposait d'une accréditation aux prérogatives jusque-là inégalées, puisqu'elle lui autorisait l'accès à la salle Louis Lumière, temple cannois s'il en est, ainsi que toute latitude pour réaliser les photographies qu'il jugeait pertinentes pour illustrer l'ouvrage Aux marches du palais (déjà cité).

15. Cf. J.-L. Fabiani, «L'offre de spectacle, la critique et la mémoire", dans E. Ethis (sous la dir. de), Avignon, le public réinventé - Le festival sous le regard des sciences sociales, Paris, La Documentation française, 2002.

L'auteur y dresse de façon saisissante le portrait d'un critique incapable de dire ce qu'il ressent face à une pièce, préférant avoir recours à ses compétences en histoire du théâtre et marquant par là même l'écart qui le sépare des spectateurs passionnés.

16. Les photographies de sosies parfaits, ceux qui peuvent être effectivement confondus avec leurs modèles illustres, doivent être classées dans les catégories étudiées plus avant, puisque comme l'affirme M. Angenot, op. cit., p. 73 : «si un simulacre présentait tous les traits du simulacre, il s'abolirait comme simulacre, du moment même où il atteindrait la perfection mimétique".

17. C. Metz, Le Signifiant imaginaire, Psychanalyse et cinéma, Paris, Christian Bourgois, 1993. Dans la croyance originaire, tous les êtres sont pourvus de pénis, alors que certains êtres sont dépourvus de pénis: c'est un témoignage de sens mais aussi un désaveu. Le fétichisme renvoie à cette castration. Le cinéma en étant la présence de l'objet par son reflet est ce «juste-avant» le désaveu que le crédule croit. Ce désaveu conditionne les clivages de la croyance chez l'homme. Le fétichisme est, dans ce que décrit Metz, l'utilisation d'un expédient, souvent figé par une pièce de vêtement et plus particulièrement un vêtement intime, qui permet de masquer l'effrayante découverte, par une fixation, de cet instant précédant le désaveu: le fétiche se substitue au pénis par métonymie.

18. R. Dulong, Le Témoin oculaire. Les conditions sociales de l'attestation personnelle, Paris, Éd. de l'École des hautes études en sciences sociales, Paris, 1998, p. 177. 